\title{
Présence
}

\section{en République Populaire du Congo \\ de Sergentomyia decipiens, Sergentomyia dissimillima, Sergentomyia tauffliebi, Sergentomyia wansoni et Sergentomyia squamipleuris}

\author{
par J. TROUILLET et G. VATTIER-BERNARD
}

(Collaboration technique: A.-S. Bimangou)

Département de Biologie animale, Equipe de Bio-écologie des Vecteurs, Faculté des Sciences, B.P. 69, Brazzaville (République Populaire du Congo).

\section{Résumé.}

Les auteurs signalent la présence en République Populaire du Congo des cinq Phlébotomes suivants : Sergentomyia decipiens Theodor, 1931; Sergentomyia dissimillima Abonnenc, 1972 ; Sergentomyia tauffliebi Abonnenc et Cornet, 1971; Sergentomyia wansoni Parrot, 1938 et Sergentomyia squamipleuris Newstead, 1912. Ils donnent la liste des Phlébotomes congolais connus au 31 mai 1976 et la carte de leur répartition géographique.

\section{Summary.}

Presence in People's Republic of Congo of Sergentomyia decipiens, Sergentomyia dissimillima, Sergentomyia tauffliebi, Sergentomyia wansoni and Sergentomyia squamipleuris.

The authors indicate the presence in People's Republic of Congo of the five following sandflies: Sergentomyia decipiens Theodor, 1931; Sergentomyia dissimillima Abonnenc, 1972; Sergentomyia tauffliebi Abonnenc and Cornet, 1971; Sergentomyia wansoni Parrot, 1938 and Sergentomyia squamipleuris Newstead, 1912. They give the check-list of sandflies of Congo on the 31st may 1976 and a map of their geographical distribution.

L'étude systématique de 23.000 Phlébotomes récoltés lors de captures quotidiennes effectuées depuis seize mois à Yaka-Yaka, village situé à $21 \mathrm{~km}$ de Brazzaville, et de récentes prospections (mars-avril 1976) dans les régions de la Bouenza et du Niari nous a permis d'identifier cinq espèces nouvelles pour la République Populaire du Congo: $S$. decipiens, $S$. dissimillima, $S$. tauffliebi, $S$. wansoni et $S$. squamipleuris.

Accepté le 11 août 1976. 


\section{Sergentomyia (Rondanomyia ?) decipiens}

Theodor, 1931

Synonymie: Phlebotomus simillimus Adler, Theodor et Parrot, 1929.

\begin{tabular}{|c|c|c|}
\hline Station de capture & Date & Piège \\
\hline Yaka-Yaka $\left(04^{\circ} 22^{\prime} \mathrm{S}-15^{\circ} 09^{\prime} \mathrm{E}\right)$ & $\begin{array}{c}1-2-75 \\
\text { au } 31-5-76\end{array}$ & $\begin{array}{l}\text { Piège adhésif lumineux } \\
\text { (Rioux et Golvan, 1969) } 42 \sigma^{\star}, 20 \text { \% }\end{array}$ \\
\hline $\begin{array}{l}\text { Au bord de la Nyanga } \\
\left(02^{\circ} 53^{\prime} \mathrm{S}-11^{\circ} 57^{\prime} \text { E) } \ldots \ldots \ldots\right.\end{array}$ & $\begin{array}{l}\text { nuit du } 27 \\
\text { au } 28-3-76\end{array}$ & 1 우 \\
\hline
\end{tabular}

Station de Yaka-Yaka.

Le village de Yaka-Yaka fait partie du plateau des Cataractes, il est entouré d'une zone de savane et se trouve distant de 4 à 500 mètres de la forêt ombrophile que traverse la rivière Djoumouna, affluent du Congo.

Avec $S$. decipiens nous y récoltons, par ordre décroissant d'importance numérique, 17 autres espèces (tableau I).

Station de la Nyanga.

La Nyanga est un fleuve qui coule dans la partie Nord-Ouest de la République Populaire du Congo et qui, se dirigeant vers le Sud-Ouest, traverse le Gabon pour aller se jeter dans l'Océan Atlantique.

Sur la berge de ce fleuve, $S$. decipiens était accompagné de :

$S$. africana africana; $S$. magna; S. dureni.

Nous avons capturé à ce jour 35.000 Phlébotomes en République Populaire du Congo (approximativement $50 \%$ en capture à main et $50 \%$ au piège adhésif lumineux). Il est intéressant de noter que nos 45 exemplaires de $S$. decipiens ont tous été récoltés au piège adhésif lumineux; ce qui nous amène à penser que nous n'avons pas découvert les gîtes de repos.

D'après Abonnenc (1972) : «S. decipiens se plaît dans les lieux couverts, d'une forte teneur en humidité. On le trouve dans les herbes des marécages, dans les cultures de bananiers, dans les forêts-galeries des savanes, le plus souvent près des points d'eau. On l'a capturé également dans les arbres creux, dans les terriers de Rongeurs. Au Zaïre, il a été capturé au Kivu dans une savane accidentée, à $1100 \mathrm{~m}$ d'altitude, à Kasenyi à $700 \mathrm{~m}$ et à Gety à $1400 \mathrm{~m}$. En République Centrafricaine, il est fréquent dans les terriers de Rongeurs. »

Répartition géographique: Zaïre, Soudan, Ouganda, Nigeria, République Centrafricaine, Côte d'Ivoire, Kenya, Guinée. 
TABleau I. - Phlébotomes capturés à Yaka-Yaka.

\begin{tabular}{|c|c|c|c|c|}
\hline Espèces & $\begin{array}{l}\text { Nombre } \\
\text { d'individus } \\
\text { capturés }\end{array}$ & $\begin{array}{c}\text { Fréquence * } \\
\text { (total } \\
\text { des captures } \\
18599 \text { ) }\end{array}$ & $\begin{array}{l}\text { Nombre } \\
\text { de relevés } \\
\text { où figure } \\
\text { l'espèce }\end{array}$ & $\begin{array}{l}\text { Constance *t: } \\
\text { (486 relevés) }\end{array}$ \\
\hline s. magna $\ldots \ldots \ldots \ldots \ldots \ldots \ldots$ & 9846 & $52,94 \%$ & 484 & $99,59 \%$ \\
\hline S. schwetzi $\ldots \ldots \ldots \ldots \ldots \ldots$ & 5256 & $28,26 \%$ & 482 & $99,18 \%$ \\
\hline S. africana africana $\ldots \ldots \ldots$ & 1768 & $9,50 \%$ & 422 & $86,83 \%$ \\
\hline S. dureni $\ldots \ldots \ldots \ldots \ldots \ldots \ldots$ & 1194 & $6,42 \%$ & 344 & $58,70 \%$ \\
\hline S. ghesquierei ............. & 163 & $0,88 \%$ & 128 & $26,34 \%$ \\
\hline S. bedfordi firmatus .......... & 139 & $0,75 \%$ & 99 & $20,37 \%$ \\
\hline Phlebotomus rodhaini $\ldots . . . .$. & 120 & $0,64 \%$ & 84 & $17,28 \%$ \\
\hline S. decipiens ............... & 61 & $0,33 \%$ & 46 & $9,46 \%$ \\
\hline S. impudica $\ldots \ldots \ldots \ldots \ldots$ & 23 & $0,12 \%$ & 21 & $4,32 \%$ \\
\hline S. collarti $\ldots \ldots \ldots \ldots \ldots \ldots$ & 14 & $0,07 \%$ & 14 & $2,88 \%$ \\
\hline S. tauffliebi $\ldots \ldots \ldots \ldots \ldots \ldots$ & 5 & $0,03 \%$ & 5 & $1,03 \%$ \\
\hline S. madagascariensis .......... & 3 & $0,02 \%$ & 3 & $0,62 \%$ \\
\hline S. bedfordi medius $\ldots \ldots \ldots \ldots$ & 2 & $0,01 \%$ & 2 & $0,41 \%$ \\
\hline S. hamoni $\ldots \ldots \ldots \ldots \ldots \ldots$ & 1 & $0,005 \%$ & 1 & $0,20 \%$ \\
\hline S. ingrami $\ldots \ldots \ldots \ldots \ldots \ldots$ & 1 & $0,005 \%$ & 1 & $0,20 \%$ \\
\hline S. lumsdeni $\ldots \ldots \ldots \ldots \ldots$ & 1 & $0,005 \%$ & 1 & $0,20 \%$ \\
\hline s. moreli $\ldots \ldots \ldots \ldots \ldots \ldots \ldots$ & 1 & $0,005 \%$ & 1 & $0,20 \%$ \\
\hline S. squamipleuris $\ldots \ldots \ldots \ldots$ & 1 & $0,005 \%$ & 1 & $0,20 \%$ \\
\hline
\end{tabular}

* La fréquence représente le pourcentage d'individus d'une espèce par rapport au total des individus.

** La constance $c$ est le rapport exprimé sous forme de pourcentage $c=\frac{\mathrm{P} \times 100}{\mathrm{P}}$ dans lequel $p$ est le nombre de relevés contenant l'espèce étudiée et $\mathbf{P}$ le nombre total de relevés effectués. (Dajoz, 1970).

\section{Sergentomyia (?) dissimillima}

Abonnenc, 1972

Synonymie: Phlebotomus simillimus inermis Abonnenc, 1958.

\begin{tabular}{|c|c|c|c|c|}
\hline Station de capture & Date & & Piège & \\
\hline 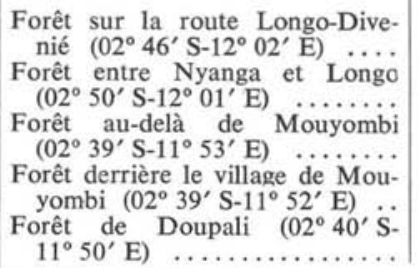 & $26-3-76$ & capture & manuelle & $\begin{array}{r}58,1 \% \\
3 q\end{array}$ \\
\hline
\end{tabular}


Nos captures ont été effectuées dans la même région (district de Divénié), en des stations proches les unes des autres, en forêt ombrophile équatoriale, sur troncs d'arbres.

Dans le même biotope, nous avons recueilli :

$S$. bedfordi medius; S. moreli; S. dyemkoumai; S. hamoni.

S. dissimillima semble avoir les mêmes habitudes que S. simillima Newstead, 1914; il paraît confiné aux régions forestières humides (Abonnenc, 1972).

Il n'avait jusqu'alors été récolté qu'en Côte d'Ivoire en 1956 avec trois points de capture : Zoanleu, Kouibly et Tiassale.

\section{Sergentomyia (Sintonius) tauffliebi}

Abonnenc et Cornet, 1971

\begin{tabular}{|c|c|c|c|c|c|c|}
\hline \multicolumn{2}{|c|}{ Station de capture } & Date & \multicolumn{4}{|c|}{ Piège } \\
\hline Yaka-Yaka & $\ldots \ldots \ldots \ldots \ldots \ldots$ & nuit du 24 & Piège & adhésif & mineux & $1 \sigma^{*}$ \\
\hline$»$ & & $\begin{array}{l}\text { au } 25-5-75 \\
\text { nuit du } 3 \\
\text { au } 4-11-75\end{array}$ & 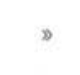 & 》 & $\gg$ & $1 \sigma^{7}$ \\
\hline$\gg$ & n.w. & $\begin{array}{l}\text { nuit du } 17 \\
\text { au } 18-11-75\end{array}$ & » & $\gg$ & 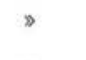 & $10^{*}$ \\
\hline 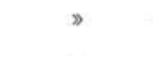 & $\cdots$ & $\begin{array}{l}\text { nuit du } 17 \\
\text { au } 18-3-76\end{array}$ & 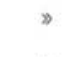 & $\gg$ & 》 & $1 \sigma^{*}$ \\
\hline 》 & n.w. & $\begin{array}{l}\text { nuit du } 23 \\
\text { au } 24-4-76\end{array}$ & 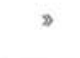 & $\$$ & $\gg$ & $1 \delta^{7}$ \\
\hline
\end{tabular}

Ce Phlébotome avait jusqu'alors été capturé en Côte d'Ivoire (1962) à Sassandra sortant d'un trou de Crabes et au Sénégal (1967 et 1969) au piège lumineux sur les rives sableuses de grands cours d'eau (Gambie et Niokolo-Koba) (Abonnenc, 1972).

Nos exemplaires, comme ceux du Sénégal, ont été capturés au piège lumineux, mais dans un biotope différent (voir précédemment). Les rives du fleuve Congo, avec berges sableuses et affleurements rocheux, qui pourraient rappeler les biotopes ivoirien et sénégalais, sont distantes de $3 \mathrm{~km}$ de notre lieu de capture.

Comme $S$. wansoni dont nous allons parler ci-après, $S$. tauffliebi appartient au sous-genre Sintonius caractérisé chez les mâles par la forme du fourreau pénien (conique court, à extrémité pointue) et chez les femelles par des spermathèques annelées. C'est la première fois que des Phlébotomes de ce sous-genre sont signalés en République Populaire du Congo. 


\section{Sergentomyia (Sintonius) wansoni}

Parrot, 1938

Synonymie: Phlebotomus matadiensis Theodor, 1938.

\begin{tabular}{|c|c|c|}
\hline Station de capture & Date & Piège \\
\hline $\begin{array}{r}\text { Grotte de Diangala }\left(03^{\circ} 58^{\prime}\right. \\
\text { S-12 } \\
\text { Rochers de Moukondo }\left(04^{\circ}\right. \\
\text { Rochers de Moukondo } \\
\left.11^{\prime} \mathrm{S}-12^{\circ} 36^{\prime} \mathrm{E}\right)\end{array}$ & 30 au $31-3-76$ & $\begin{array}{l}\text { Capture manuelle } 4 \delta^{*} \\
\text { Pièges adhésifs } 3 \delta, 1 \%\end{array}$ \\
\hline
\end{tabular}

\section{Station de Diangala.}

La grotte de Diangala est située dans un éperon rocheux qui domine la savane de la région de Mouindi, elle est peu profonde, il s'agit d'ailleurs plutôt d'un abri sous roche. Nous y avons capturé quatre exemplaires de $S$. wansoni au voisinage de nids d'Oiseaux (non identifiés). Ils n'étaient accompagnés d'aucun représentant d'autres espèces.

\section{Station de Moukondo.}

Les rochers de Moukondo, en bordure de la route du Mayombe, se trouvent aussi dans une région de savane. Nous avons capturé dans ce biotope:

- à la main : S. africana africana uniquement ;

- avec des pièges adhésifs: $S$. wansoni, $S$. africana africana, $S$. collarti.

Répartition géographique: Matadi (Zaïre), Koutiala (Mali), Aloro (Ouganda), Dundo (Angola).

\section{Sergentomyia (Grassomyia) squamipleuris}

Newstead, 1912

Synonymie: (?) Phlebotomus iraqi Adler et Theodor, 1929;

Phlebotomus horgani Lewis et Kirk, 1946 pro parte.

\begin{tabular}{|c|c|c|c|}
\hline Station de capture & Date & Piège & \\
\cline { 2 - 2 } Yaka-Yaka ................. & nuit du 6 au 7-10-75 & Piège adhésif lumineux & $1 \%$ \\
\hline
\end{tabular}


Dans une révision du sous-genre Grassomyia, Abonnenc (1969) a montré que la variation de la vestiture écailleuse des pleures thoraciques et notamment celle des mésanépimères pouvait servir à séparer les cinq espèces de ce sous-genre, connues en région éthiopienne.

En République Populaire du Congo, nous avons jusqu'à maintenant récolté trois de ces espèces : $S$. ghesquierei, $S$. madagascariensis et $S$. squamipleuris.

S. ghesquierei se distingue des deux autres par l'absence d'écailles sur les mésanépimères thoraciques des deux sexes. Nos exemplaires de $S$. madagascariensis comptent 4 à 15 écailles mésanépimérales pour les mâles, 3 à 12 pour les femelles (VattierBernard et Trouillet, 1976. La femelle de $S$. squamipleuris que nous avons capturée présente 30 écailles mésanépimérales.

Les Phlébotomes de ce sous-genre sont fortement attirés par les lumières (Abonnenc, 1972). Sur les 235 individus que nous avons récoltés, 217 ont été capturés par piégeage lumineux, 18 à la main dont 17 sur troncs d'arbres et un seul sur un mur d'une case habitée.

Répartition géographique: Zaïre, Dahomey, Ethiopie, Gambie, Guinée, HauteVolta, Kenya, Mali, Mozambique, Nigeria, Ouganda, République Centrafricaine, Sénégal, Soudan, Tanzanie, Tchad.

\section{Liste des Phlébotomes congolais}

Avec ces cinq nouvelles espèces, la liste des Phlébotomes congolais se compose de 35 espèces et sous-espèces appartenant à trois genres et à huit sous-genres (fig. 1).

\section{Genre Phlebotomus:}

1) Phlebotomus (Anaphlebotomus) rodhaini Parrot, 1930.

Genre Spelaeophlebotomus :

2) Spelaeophlebotomus gigas Parrot et Schwetz, 1937.

Genre Sergentomyia :

a) sous-genre Grassomyia.

3) Sergentomyia (Grassomyia) ghesquierei Parrot, 1929.

4) Sergentomyia (Grassomyia) madagascariensis Abonnenc, 1969.

5) Sergentomyia (Grassomyia) squamipleuris Newstead, 1912.

b) sous-genre Spelaeomyia.

6) Sergentomyia (Spelaeomyia) emilii Vattier, 1966.

7) Sergentomyia (Spelaeomyia) mirabilis Parrot et Wanson, 1939.

8) Sergentomyia (Spelaeomyia) moucheti Vattier-Bernard et Abonnenc, 1967.

c) sous-genre Sergentomyia.

9) Sergentomyia (Sergentomyia) bedfordi congolensis Bequaert et Walravens, 1930. 


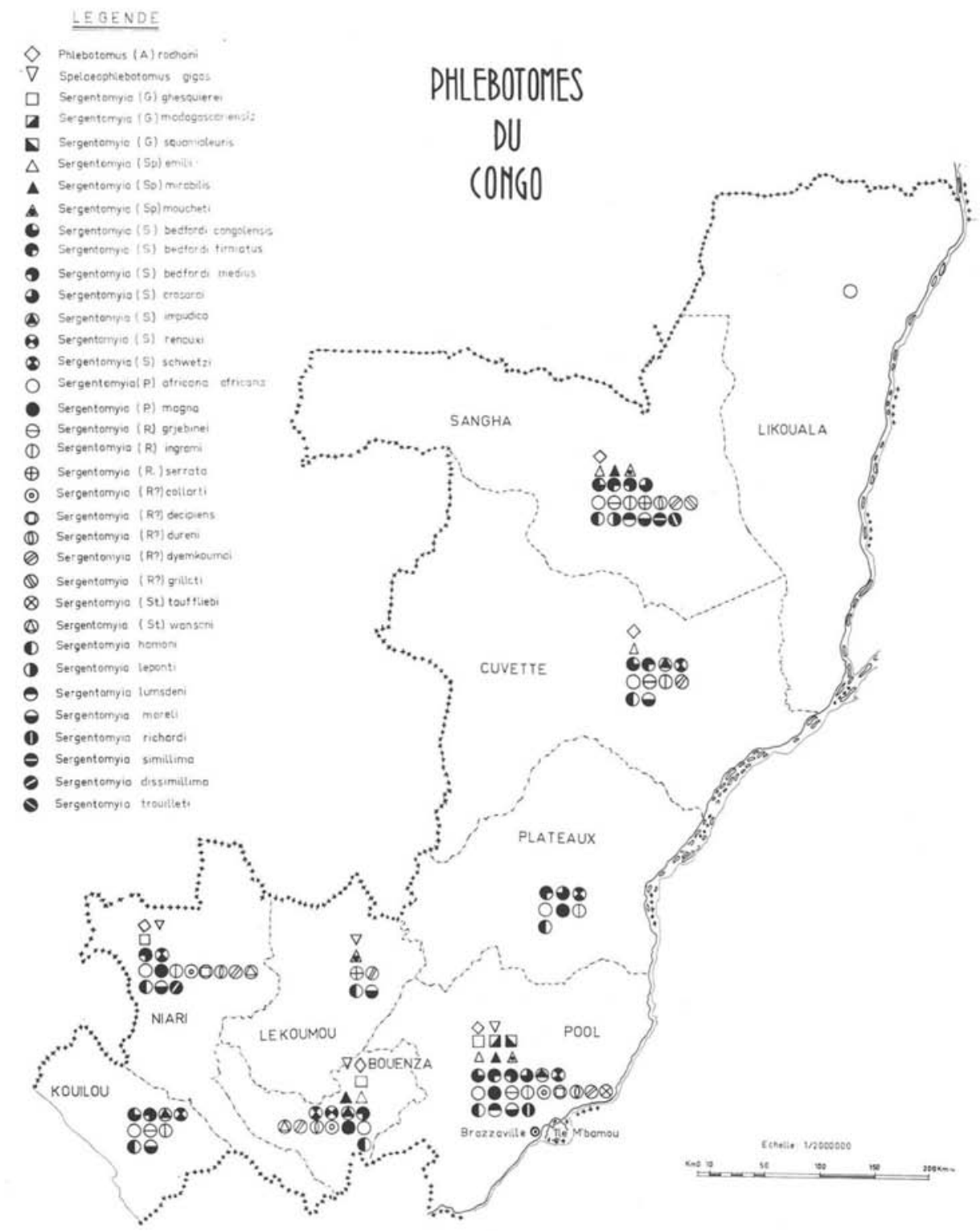

FIG. 1. - Répartition des Phlébotomes congolais. 
10) Sergentomyia (Sergentomyia) bedfordi firmatus Parrot et Malbrant, 1945.

11) Sergentomyia (Sergentomyia) bedfordi medius Kirk et Lewis, 1950.

12) Sergentomyia (Sergentomyia) crosarai Parrot et Wanson, 1946.

13) Sergentomyia (Sergentomyia) impudica Abonnenc, 1968.

14) Sergentomyia (Sergentomyia) renauxi Parrot et Schwetz, 1937.

15) Sergentomyia (Sergentomyia) schwetzi Adler, Theodor et Parrot, 1929.

d) sous-genre Parrotomyia.

16) Sergentomyia (Parrotomyia) africana africana Newstead, 1912.

17) Sergentomyia (Parrotomyia) magna Sinton, 1932.

e) sous-genre Rondanomyia.

18) Sergentomyia (Rondanomyia) grejbinei Vattier-Bernard, 1971.

19) Sergentomyia (Rondanomyia) ingrami Newstead, 1914.

20) Sergentomyia (Rondanomyia) serrata Parrot et Malbrant, 1945.

21) Sergentomyia (Rondanomyia?) collarti Adler, Theodor et Parrot, 1929.

22) Sergentomyia (Rondanomyia ?) decipiens Theodor, 1931.

23) Sergentomyia (Rondanomyia?) dureni Parrot, 1934.

24) Sergentomyia (Rondanomyia?) dyemkoumai Abonnenc, 1964.

25) Sergentomyia (Rondanomyia ?) grilloti Vattier-Bernard, 1975.

f) sous-genre Sintonius.

26) Sergentomyia (Sintonius) tauffliebi Abonnenc et Cornet, 1971.

27) Sergentomyia (Sintonius) wansoni Parrot, 1938.

g) Incertae sedis.

28) Sergentomyia hamoni Abonnenc, 1958.

29) Sergentomyia leponti Vattier-Bernard, 1973.

30) Sergentomyia lumsdeni Kirk et Lewis, 1950.

31) Sergentomyia moreli Abonnenc et Hamon, 1958.

32) Sergentomyia richardi Parrot et Wanson, 1946.

33) Sergentomyia simillima Newstead, 1914.

34) Sergentomyia dissimillima Abonnenc, 1972.

35) Sergentomyia trouilleti Vattier-Bernard, 1976.

\section{Bibliographie}

ABonnenc (E.), 1969. - Sur Phlebotomus squamipleuris Newstead, 1912 et quelques espèces voisines (Diptera, Psychodidae). Cah. O.R.S.T.O.M., sér. Entomol. Méd. Parasitol., 8, 307-323.

Abonnenc (E.), 1972. - Les Phlébotomes de la région éthiopienne (Diptera, Psychodidae). Mém. O.R.S.T.O.M., 55, 289 p. 
Dajoz (R.), 1970. - Précis d'écologie. Dunod, édit., Paris, 357 p.

Vattier-Bernard (G.), 1973. - Phlebotomus (Sergentomyia) leponti (Diptera, Psychodidae). Espèce nouvelle récoltée au nord de la République Populaire du Congo. Cah. O.R.S.T.O.M., sér. Entomol. Méd. Parasitol., 11, 293-294.

Vattier-Bernard (G.) et Bimangou (A.-S.), 1975. - Phlebotomus (Sergentomyia) grilloti n. sp. (Diptera, Psychodidae), espèce nouvelle récoltée dans le nord du Congo. Cah. O.R.S.T.O.M., sér. Entomol. Méd. Parasitol., 13, 117-119.

Vattier-Bernard (G.) et Trouillet (J.), 1976. - Phlebotomidae (Diptera) du sous-genre Grassomyia en République Populaire du Congo. Cah. O.R.S.T,O.M,, sér. Entomol. Méd. Parasitol., 14, 83-87.

VAtTier-Bernard (G.), 1976. - Sergentomyia trouilleti (Diptera, Phlebotomidae), espèce: nouvelle récoltée au Congo. Ann. Parasitol. hum. comp., 51, 667-671. 